\title{
The Galactic Center
}

\author{
Farhad Yusef-Zadeh \\ Northwestern University \\ Dept. Physics and Astronomy \\ Evanston, Illinois 60208, USA
}

\begin{abstract}
The dynamic subject of the Galactic center is driven mostly by high-resolution imaging both in the continuum and in the spectral line modes in a number of wavelength regimes. An attempt has been made to give a short overview of recent observations in this region, as follows: 1) Unusual characteristics of the population of molecular and dust clouds within the inner few hundred pc of the Galactic center. 2) Large-scale magnetic structures within the inner $100 \mathrm{pc}$ of the Galactic center and the possible evidence for their poloidal configurations -unlike that seen in the disk of the Galaxy. 3) Stellar kinematics in the bulge and the possible evidence for a massive central source at the dynamical center and its examination as a gravitational lens. 4) Morphological and kinematic evidence for the Galactic center wind interacting with stellar wind associated IRS7, a mass-losing supergiant lying one light year away in projection from the Galactic center.
\end{abstract}

\section{Introduction}

The study of the Galactic center has a unique place in the history of Astronomy in that it coincides with the birth of radio Astronomy when Jansky first reported the detection of radio emission from the Galactic center in 1933. The investigation of this region has generally been tied to the development of new instrumentations (e.g. large-format near-IR spectrometers, VLBA, SIGMA telescope on board GRANAT sattelite, and GRO) because it is a strong radio and infrared source, and is a closest example of a Galactic nucleus. This region of the Galaxy is unique in that it is the only Galactic nucleus for which an analysis of the motion of the stars and gas has been made. Furthermore, the Galaxy's spiral density wave does not penetrate this region, so a comparison of star formation activity with that in the spiral arms would prove interesting.

Since the extinction of light by dust is greater than 28 magnitudes, much of our understanding of this region comes from observational clues revealed in the lowenergy domain beyond $\lambda 1 \mu \mathrm{m}$ (see reviews by Brown and Liszt 1984 and Genzel and Townes 1987), although there is a recent surge of very important space-based highenergy observations on the nature of $511 \mathrm{KeV}$ emission from this region (Sunyaev $e t$ al. 1991; Bouchet et al. 1991). Because of this restriction in the visual wavelengths, accurate information about stellar components is not yet available. In lower energy domains, an enormous complexity associated with numerous components with different scale sizes have been recognized. In spite of this complexity, it is still possible 
to produce hypotheses about general physical conditions and about a wide range of phenomena that are present in the nucleus.

\section{II.Global Characteristics}

On a 500pc scale, the Galactic center region interstellar medium is dominated by the molecular gas. About $10 \%$ of the Galaxy's neutral gas is estimated to lie within a few hundred pes of the Galaxy. The population of molecular clouds differs from those in the disk of the Galaxy. The molecular clouds in this region are pervasively massive $\left(10^{5}-10^{6} \mathrm{M}_{\odot}\right)$, and large $(20-30 \mathrm{pcs})$ in size having higher velocity dispersions $(10-30 \mathrm{~km} / \mathrm{s})$, higher kinetic temperature $(\approx 70 \mathrm{~K})$ and denser $\left(\approx 10^{4} \mathrm{~cm}^{-3}\right)$ than in other parts of the Galaxy. The high density of molecular gas in this region is a consequence of a steep gravitational potential which allows only the high-density gas to be gravitationally bound (Bally et al. 1988; Güsten 1989). These clouds have to be dense enough to excite CS $(2 \rightarrow 1)$ line toward two prominent sources, Sgr B1 and Sgr B2, as shown in Figure 1. In spite of such a reservoir of dense molecular gas, the efficiency of star formation rate appears to be low when compared to spiral arms of regions (Güsten 1989; Morris 1989). Recently, Stark et al. (1991) have considered the possibility that these massive gas clouds in the central kpc experience a significant frictional drag from the star in the bulge. Consequently, the gas clouds should spiral to the center over a dynamical friction time scale, $\tau_{f} \approx 4 \times 10^{8} \mathrm{yrs}$, which implies that that the present population of the Galactic center clouds are transient (Stark et al. 1991).

Most of the luminosity from the Galactic center region is seen in the far-IR. The total bolometric luminosity from the inner $3^{0} \times 2^{0}$ is estimated to be $\approx 10^{9} L_{\odot}$ and the dust temperature varies between $23-35 \mathrm{~K}$ with a $\mathbf{r}^{-0.3}$ radial dependence (Cox and Laureijs 1989). The infrared excess of extended emission in this region may result from absorption of stellar Lyman photons from a population of late-type of stars.

\section{Magnetic Phenomena}

On a scale of tens of pcs, ordered magnetic fields are seen along a variety of narrow filaments in a number of locations in the Galactic center region (Yusef-Zadeh 1989). Some of these synchrotron emitting features are remarkably rigid and remain linear over many tens of pcs. Figure 2a shows the latest example of such a magnetic feature near the Galactic center. This remarkable feature, G359.1-0.2, which extends over $15^{\prime}$ or $\approx 38 \mathrm{pc}$ is distinguished from other nonthermal filaments in that it shows a number of kinks (Gray et al. 1991). The southeastern tail end of this feature is also seen Figure 1b. The nature of these nonthermal filaments remain mysterious in spite of the fact that there are a number of MHD theories that have attempted to explain their structure. Some of these theories involve induced large scale currents and other involve expansion of magnetic loops from the disk or from the dynamical center of the Galaxy (Benford 1989; Heyvaerts et al. 1988; Shibata and Uchida 1987). 


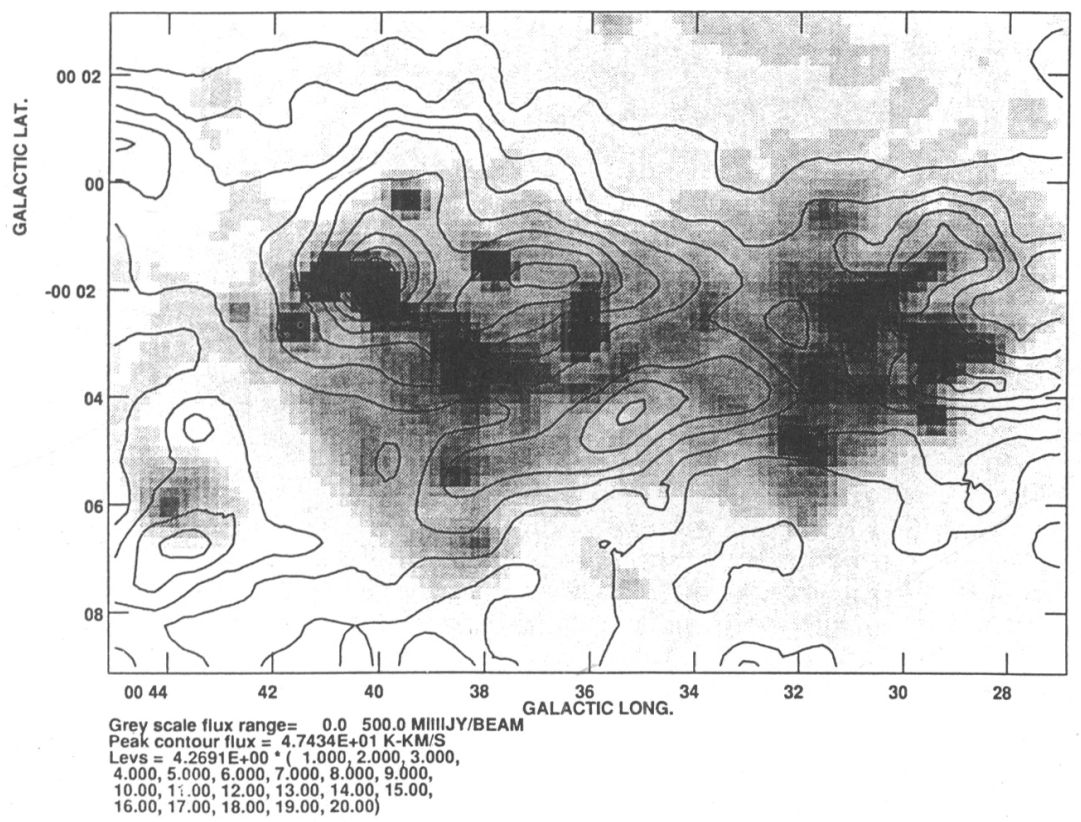

Figure 1: Contour map of integrated CS lines between 78 and $123 \mathrm{~km} / \mathrm{s}$ using SEST telescope is superimposed on a gray scale continuum map of Sgr B1 and Sgr B2 (Yusef-Zadeh et al. 1991).

The most prominent of the network of magnetic systems is known as the radio continuum Arc at $1 \approx 0.2^{0}$ which consists of thermal and nonthermal features. On morphological grounds, arguments have been made that these two features interact with each other. However, hard evidence for such a physical interaction and the possible role of MHD processes in ionizing thermal features are lacking (Morris and Yusef-Zadeh 1989; Genzel et al. 1990). The direction of the magnetic field associated with the Arc and its large-scale extensions appear to be predominantly in the direction perpendicular to the Galactic plane (Tsuboi et al. 1986). This has lead to the suggestion that the physics of the interstellar medium in the Galactic center region may be dominated by the poloidal component of the magnetic field. There are currently a number of far-IR and radio measurements being carried out toward thermal features and nonthermal filaments in the Arc to determine the strength and the intrinsic direction of the magnetic field. If the magnetic field pressure associated with these features is large enough to be important dynamically, it is possible that accretion could be driven toward the black hole candidate at the Galactic center by the removal of angular momentum.

On a scale of about 10pc, a nonthermal shell-like source known as Sgr A East, is seen toward the well-known ionized source, Sgr A West which engulfs the center of 

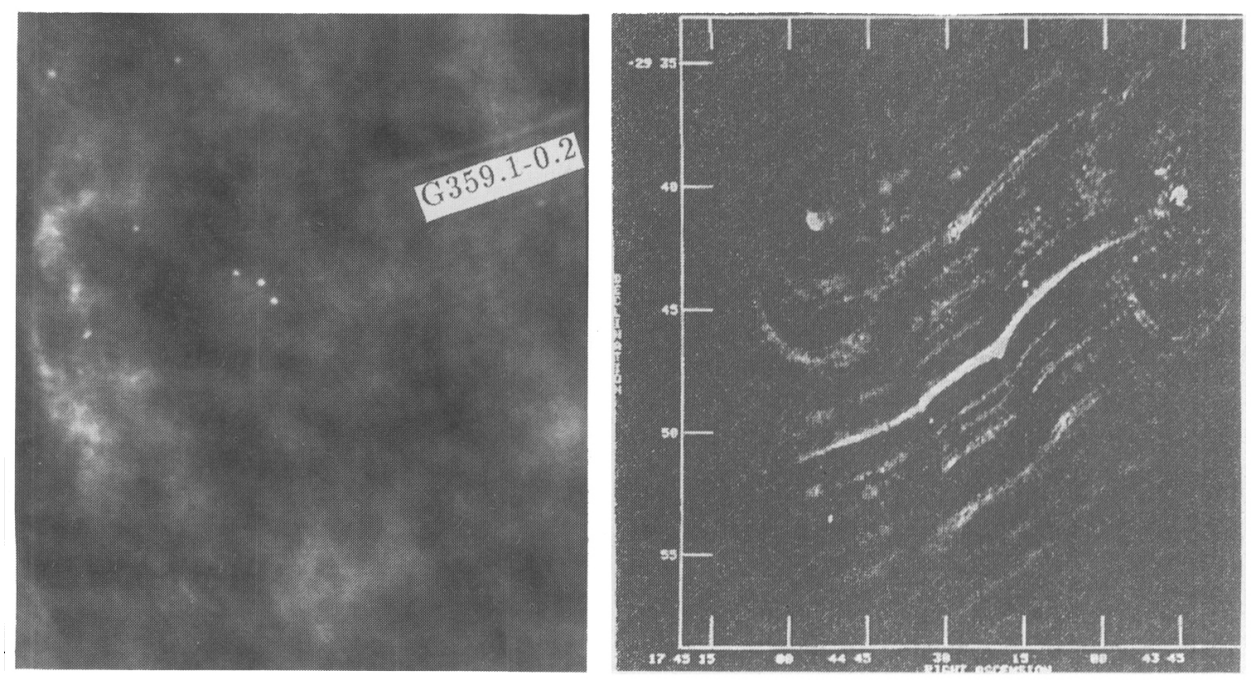

Figure 2a,b: The image to the right (Fig. 2b) shows G359.1-0.2 filaments at $\lambda 6 \mathrm{~cm}$ based on ATCA observations (Gray et al. 1991). The image to the left shows the $\lambda 20 \mathrm{~cm}$ image of the southeastern segment of G359.1-0.2 filament using the VLA in its $\mathrm{B} / \mathrm{C}$ configuration.

the Galaxy. There has been considerable confusion as to the relative location of this source with respect to Sgr A West whose proximity to the Galactic center is well established. The position of this feature is recognized beautifully in recent $327 \mathrm{MHz}$ maps where Sgr A West becomes increasingly more opaque at longer frequencies and consequently absorbs the background radiation associated with Sgr A East. At low frequencies, $\mathrm{Sgr}$ A West has totally disapeared, as shown in Figure 3 (Pedlar et al. 1989). This geometry has confirmed earlier conclusion based on measurements made using Culgoora Radioheliograph (Yusef-Zadeh and Morris 1987). Another geometrical aspect of Galactic center features is related to the relative position of the 20 and $40 \mathrm{~km} / \mathrm{s}$ molecular clouds with respect to the Galactic center. Geballe $e t$ al. (1991) have used absorption spectra of low-lying fundamental vibration-rotation bands of $\mathrm{CO}$ at $4.64 \mu \mathrm{m}$ to infer that the $20 \mathrm{~km} / \mathrm{s}$ is situated in front of the Galactic center whereas the $40-60 \mathrm{~km} / \mathrm{s}$ clouds located behind most of Sgr A West.

IV. Sgr A*, IRS16 and IRS7 

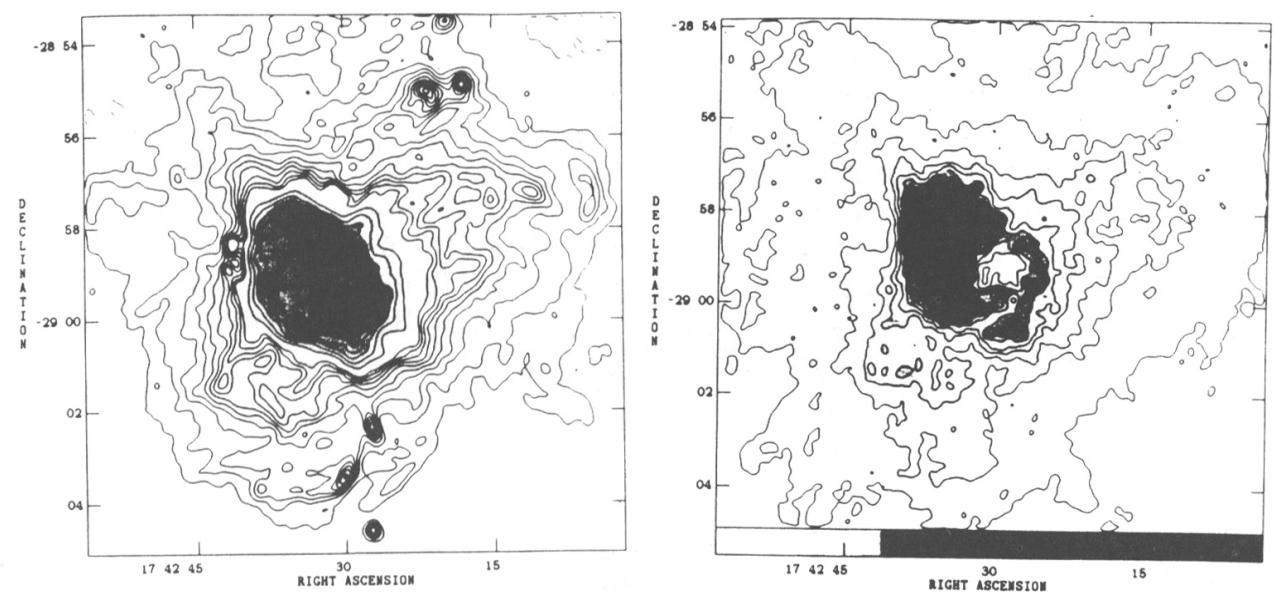

Figure 3a,b: The image to the left shows Sgr A East and West at $\lambda 20 \mathrm{~cm}$. The same region is shown at $\lambda 90 \mathrm{~cm}$ to the right (Pedlar et al. 1989).

Perhaps one of the most enigmatic objects found in the Galactic center region is the compact radio source, known as $\mathrm{Sgr} \mathrm{A}^{*}$. This variable source (Zhao et al. 1989) shows nonthermal characteristics with a spectrum which resembles the cores of extragalactic radio sources (1989). With a radio luminosity of $\sim 2 \times 10^{34} \mathrm{erg} / \mathrm{s}$, $\mathrm{Sgr} \mathrm{A}^{*}$ is the brightest radio source within the inner few degrees of the Galactic center region. The characteristics of $\mathrm{Sgr} \mathrm{A}^{*}$ make it an unusual source, leading some to consider it as the candidate for the $10^{3} \mathrm{M}_{\odot}$ (Allen and Sanders 1986) to several times $10^{6} \mathrm{M}_{\odot}$ black hole (Lacy et al. 1991; McGinn et al. 1989; Sellgren et al. 1990). Its identification as a massive black hole is also suggested indirectly by its low proper motion, $\leq 40 \mathrm{~km} \mathrm{~s}^{-1}$ (Backer and Sramek 1986). The case for such a massive black hole there has also been inferred by detailed examination of the velocity field of ionized gas in Sgr A West orbiting the Galactic center with the assumption that non-gravitational forces, such as magnetic forces, are not important in influencing the gas dynamics. Sgr A* lies near the dynamical center of an evolved stellar cluster. The underlying stellar density profile shows a dependence on radius $\mathbf{r}^{-1.8}$ over three orders of magnitudes in r. McGinn et al. (1989) and Sellgren et al. (1990) used observations of the integrated $2.3 \mu \mathrm{m}$ bandhead of $\mathrm{CO}$ toward late-type cluster stars to determine the kinematics of the cluster and inferred the presence of concentrated dark matter at the dynamical center of the Galaxy.

The presence of a massive black hole may be detected by the gravitational lensing of Sgr A East, a nonthermal radio source lying behind the Galactic center which is 
thought to be within the inner kpc of the Galaxy (Yusef-Zadeh and Morris 1987; Pedlar et al. 1989). Substructures associated with Sgr A East are recognized to lie at a projected distance $\theta_{1} \approx 10^{\prime \prime}$ away from the Galactic center (Pedlar et al. 1989), so a secondary image should lie at $\approx 3.6$ milliarcsecond if Sgr A East is 100 pc away. Surface brightness is conserved by lensing, so that given the morphology of Sgr A East, one may predict the appearance of the secondary image. By way of example, Figure 4 shows the image of a very simple representation of Sgr A East (Wardle and Yusef-Zadeh 1991). Indeed, there is already evidence for extended structure near $\mathrm{Sgr} \mathrm{A}^{*}$ on a 20 milliarcsecond scale at $\lambda 6 \mathrm{~cm}$ (R.L. Brown, private comm.)

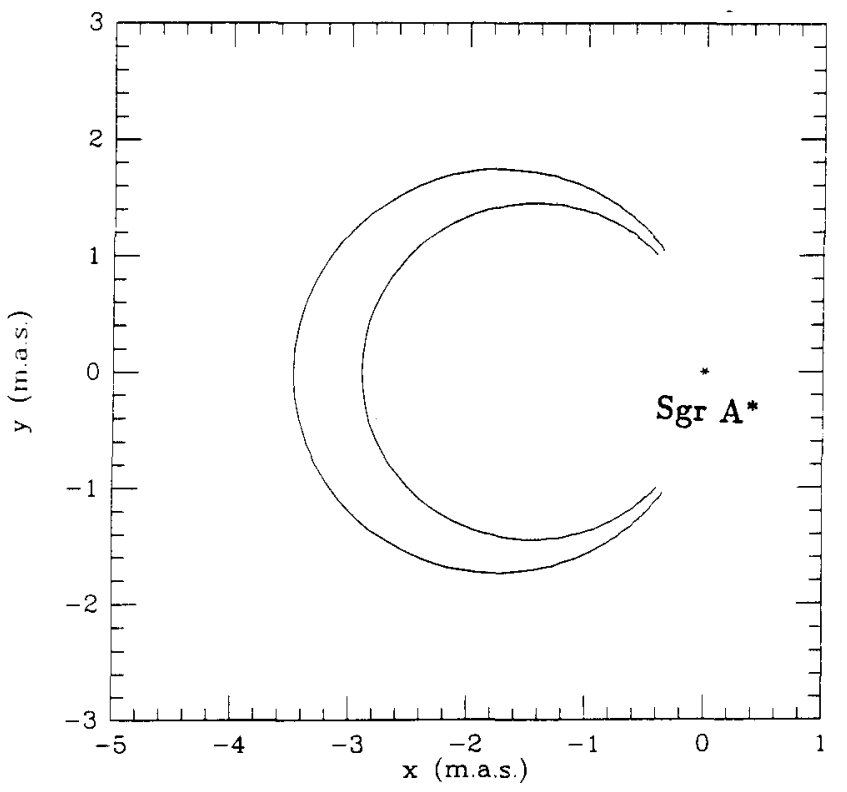

Figure 4: A schematic illustration of the lensing of Sgr A East by a $10^{6} \mathrm{M}_{\odot}$ black hole at the position of $\operatorname{Sgr} \mathrm{A}^{*}$. A segment of Sgr A East was represented by two vertical lines $10^{\prime \prime}$ away from $\mathrm{Sgr} \mathrm{A}^{*}$.

Another unusual bright infrared source-known as IRS16-lying within $\sim 1^{\prime \prime}$ of Sgr $A^{*}$ is surrounded by a cluster of late type stars and has occasionally been cited as the key energy source at the Galactic center. IRS16 lies within the inner $1^{\prime \prime}$ of the dynamical center of the Galaxy and consists of a number of infrared components, all of which have blue colors relative to luminous evolved stars. Allen et al. (1990) have pointed out that the line emission arising from IRS16 subcomponents has a spectrum which is consistent with that of mass-losing WN stars. The case for such a wind has been strenghtened recently by detection of ionized gas from IRS7, a supergiant within the projected distance of one light year (Rieke and Rieke 1989; Yusef-Zadeh and Morris 1991; Serabyn, Lacy and Achtermann 1991). It is natural to 
cosider the interaction of the Galactic wind from IRS16 sources and the outflow from IRS7. Figure 5 shows the bow shock structure of IRS7 resulting from two colliding winds. Superimposed on the radio map of Figure 2 is the simulation of the surface of the bow drawn to the same scale, for 4 different values of the parameter $F$ ( $F^{2}$ is defined as the ratio of the momentum rate of the Galactic wind to that of stellar wind) (Yusef-Zadeh and Melia 1991). The location of IRS7 is determined solely from the fit of the bow shape, and it is noteworthy that its theoretical positioning is consistent with that due to near-IR astrometric measurements of IRS 7 .

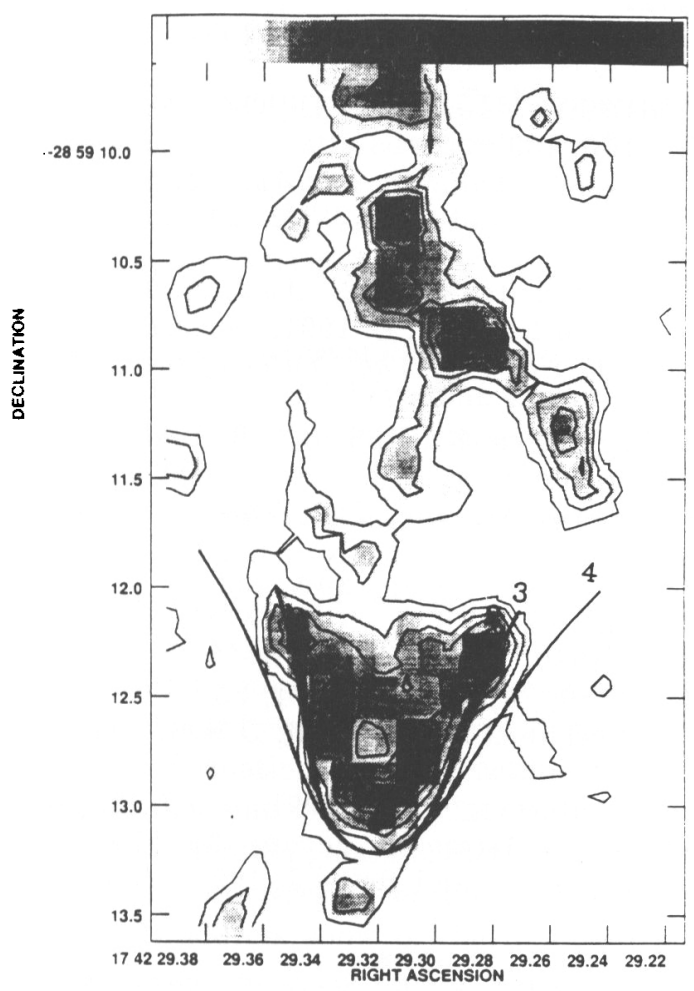

Figure 5: Plot of outer shock radius for 4 different theoretical values of F, 500, 100, 50,10 corresponding to plots $1,2,3,4$ superimposed on the bow-shock strcuture of IRS7. (Yusef-Zadeh and Melia 1991).

I wish to thank Mark Wardle for his critical reading of this manuscript.

\section{References}

Allen, D.A., Hyland, A.R. and Hillier, D.J. 1990, Mon.Not.R.astr.Soc., 244, 706. 
Allen, D.A., and Sanders, R.H. 1986, Nature, 319, 191.

Backer, D.C. and Sramek, R.A. 1982, Ap.J., $260,512$.

Bally, J., Stark, A., Wilson, R. and Henkel, C. 1988, Ap.J., 324, 23.

Benford, G. 1988, A p.J. 333, 735 .

Bouchet, L. et al. 1991, A p.J.Lett., in press.

Brown, R.L. and Liszt, J.H. 1984, Ann.Rev.Astron.Astrophys., 22,223 .

Cox, P. and Laureijs, R. 1989, IAU Symposium No. 136, The Center of the Galaxy, ed. M. Morris, p121.

Geballe, T.R., Krisciunas, K., Bailey, J.A., Wade, R. 1991, Ap.J.Lett. $\mathbf{3 7 0 , L 7 3 .}$

Genzel, R., Stacey, G.J., Harris, A.I., Townes, C.H., Geis, N., Graf, U.U. et al. 1990, Ap.J., 356, 160.

Genzel, R. and Townes, C.H. 1987, Ann.Rev.Astr.Ap., 25, 377.

Gray, A.D., Cram, L.E., Ekers, R.D. and Goss, W.M. 1991, Nature, $\mathbf{3 5 3}, 237$

Ileyvaerts, J., Norman, C. and Pudritz, R. 1988, Ap.J., 330, 718.

Lacy, J.H., Achtermann, J.M. and Serabyn, E. 1991, Ap.J.Lett., in press.

Lo, K.Y. 1989, IAU Symposium No. 136, The Center of the Galaxy, ed. M. Morris, p 527 .

McGinn, M.T., Becklin, E.E., Sellgren, K., Hall, D.N.B. 1989, Ap.J., $\mathbf{3 3 8}, 824$.

Morris, M. 1989, IAU Symposium No. 136, The Center of the Galaxy, ed. M. Morris, p171.

Morris, M. and Yusef-Zadeh, F. 1989, Ap.J., 343, 703.

Pedlar, A., Anatharamiah, K.R., Ekers, R.D., Goss, W.M., van Gorkom, J.H., Schwarz, U.J., and Zhao, J. 1989, Ap.J., 342, 769.

Rieke, G.II. and Rieke, M.J. 1989, Ap.J.Lett., 344, L5.

Sellgren, K., McGinn, M.T., Becklin, E.E. and Hall, D.N.B. 1990, Ap.J., 359,312 .

Serabyn, E., Lacy, J.H., and Achtermann, J.M. 1991, Ap.J., in press.

Shibata, K. Uchida, Y. 1987, Publ.Astron.Soc.Japan, 39, 559.

Stark, A.A., Gerhard, O.E., Binney, J. and Bally, J. 1991, MNRAS, $248,14 \mathrm{p}$.

Sunyaev, L. et al. 1991, Ap.J.Lett., in press.

Tsuboi, M. Inoue, M., Handa, T., Tabara, H. Kato, T., Sofue, Y. and Kaifu, N. 1986, Astron.J., $\mathbf{9 2 , 8 1 8 .}$

Wardle, M. and Yusef-Zadeh 1991, in preparation.

Yusef-Zadeh, F. 1989, IAU Symposium No. 136, The Center of the Galaxy, ed. M. Morris, p243.

Yusef-Zadeh, F. et al. 1991, in preparation.

Yusef-Zadeh, F., Melia, F. 1991, Ap.J.Lett., submitted.

Yusef-Zadeh, F. and Morris, M. 1987, Ap.J., 322, 721.

- - 1991, Ap.J.Lett., 371, L59.

Zhou, J.H., Ekers, R., Goss, M., Lo, K.Y. Narayan, R. 1989, IAU

Symposium No. 136, The Center of the Galaxy, ed. M. Morris, p535. 